\title{
Utility of Nebulized Magnesium Sulfate Therapy for Persistent Pulmonary Hypertension of Newborn
}

\author{
Adhisivam Bethou' ${ }^{1}$
}

Received: 7 May 2021 / Accepted: 8 June 2021 / Published online: 23 June 2021

(c) Dr. K C Chaudhuri Foundation 2021

Persistent pulmonary hypertension of the newborn (PPHN) occurs primarily in term or late preterm neonates and is associated with increased pulmonary vascular resistance after birth, causing right-to-left shunting of blood via fetal circulatory pathways. Underdevelopment, maldevelopment, or maladaptation of the pulmonary vasculature may be the basic pathologyin PPHN [1]. Supplemental oxygen, mechanical ventilation, correction of acidosis, appropriate fluid therapy and inotropic agents are usual strategies for managing PPHN. Apart from supportive care, sildenafil, inhaled nitric oxide, and extracorporeal membrane oxygenation are established treatment options for PPHN. In resourcerestricted settings, these expensive therapies are often not feasible. Magnesium sulfate (MS) is an effective vasodilator, and hence may reduce high pulmonary arterial pressure in PPHN. If MS is found useful for treating PPHN, it would possibly be a cost-effective and lifesaving therapy. A Cochrane review by Ho and Rasa (2007) did not find enough evidence for the use of MS in PPHN and the authors had recommended randomized, controlled trials (RCTs) [2]. In this context, the pilot randomized controlled trial by Abdelkreem et al. gains importance [3].

This pilot RCT from Egypt had included 28 term neonates with severe PPHN who were on ventilator. These neonates were randomized equally to 2 groups namely, NebMag group — who were administered nebulized isotonic MS and IVMag group — who were administered intravenous MS. Oxygenation index (OI), mean arterial blood pressure (MABP), vasoactive inotropic score (VIS), and magnesium in serum were measured over $24 \mathrm{~h}$. OI reduced by $44.3 \%$ and $35.3 \%$ in the NebMag group and IVMag group, respectively. The NebMag group had better MABP and lesser VIS at $24 \mathrm{~h}$.

Adhisivam Bethou

adhisivam1975@yahoo.co.uk

1 Department of Neonatology, Jawaharlal Institute of Postgraduate Medical Education and Research (JIPMER), Pondicherry 605 006, India
The rise in serum magnesium concentration assessed at $12 \mathrm{~h}$ was lesser in the NebMag group [3]. Intention to treat analysis has been done though not specified.

Antenatal administration of MS to women who are prone for very preterm birth is an effective fetal neuroprotective intervention. It has definite utility in the management of eclampsia and severe acute asthma. The role of MS as an adjunct to therapeutic hypothermia and persistent pulmonary hypertension of newborn is also being explored. MS may be useful in PPHN by its sedative, muscle relaxant, and bronchodilator effects or associated alkalosis [4]. The usual side effects for MS include vomiting, flushing, thirst, hypotension, drowsiness, confusion, loss of deep tendon reflexes, muscle weakness, respiratory depression, and cardiac arrhythmias. In neonates, it appears to be relatively safe in the usual dose, especially when given in the nebulized form. This RCT has also not mentioned any significant side effects of MS like hypotension.

Rapid onset of action and decreased side effects are the advantages when MS is administered by nebulization. However, decreased quantity of drug delivered compared to intravenous preparation and increased respiratory effort of the patient for improving drug delivery are the potential disadvantages. Several drugs including bronchodilators, steroids, surfactant, and antibiotics may be administered during mechanical ventilation using nebulizers. Aerosolized drug administration from a nebulizer to the distal airways of a mechanically ventilated patient can be affected by the type of nebulizer used, residual volume, mode of nebulization, position of the nebulizer in the ventilator circuit, and gas flow in the circuit. These practical issues can affect the dose delivered, and hence, comparisons of clinical outcomes may not be accurate.

The authors have themselves mentioned the limitations of this RCT. This study was not prospectively registered with www.clinicaltrials.gov. The sample size was small and almost $65 \%$ of the neonates have been excluded after initial screening. Even though this may be a pilot study, an attempt 
for a reasonable sample size calculation could have been made from a similar study done in the same NICU [5]. Offsite randomization is always better and details regarding who administered the intervention and who recorded the outcome could have been made explicit. No deaths were recorded during the study period of $24 \mathrm{~h}$. However, deaths could have occurred 24-72 $\mathrm{h}$ after the intervention. Hence, need for repeat doses and longer duration of follow-up should be considered in future studies. The utility of the same intervention i.e., using nebulized MS for PPHN in nonventilated neonates should also be explored.

Despite all the above limitations, this study is a small step towards seeking evidence for improving neonatal intensive care. This study does not give concrete evidence for nebulized MS utility for treating mechanically ventilated neonates with severe PPHN. However, seemingly off-label drugs may also be incorporated in standard protocols after gaining enough evidence through continued stringent research.

\section{References}

1. Chawla D. Vasopressin in persistent pulmonary hypertension of newborn. Indian J Pediatr. 2021;88:431-2.

2. Ho JJ, Rasa G. Magnesium sulfate for persistent pulmonary hypertension of the newborn. Cochrane Database Syst Rev. 2007; CD00558. https://doi.org/10.1002/14651858.CD005588.

3. Abdelkreem E, Mahmoud SM, Aboelez MO, Abd El Aal M. Nebulized magnesium sulfate for treatment of persistent pulmonary hypertension of newborn: a pilot randomized controlled trial. Indian J Pediatr. 2021. https://doi.org/10.1007/s12098-02003643-y.

4. Patole SK, Finer NN. Experimental and clinical effects of magnesium infusion in the treatment of neonatal pulmonary hypertension. Magnes Res. 1995;8:373-88.

5. Abdalsalam AA, Mahmoud RA, Mohamed MA, Hadhoud SE. Inhalational versus intravenous magnesium sulfate in management of pulmonary hypertension in neonates. Sohag Med J. 2020;24:1-13.

Publisher's Note Springer Nature remains neutral with regard to jurisdictional claims in published maps and institutional affiliations.

\section{Declarations}

Conflict of Interest None. 